\title{
Quantifying Magnetism on the nm Scale: EMCD on Individual FePt Nanoparticles
}

Sebastian Schneider ${ }^{1,2}$, Darius Pohl ${ }^{1}$, Stefan Löffler ${ }^{3,4}$, Deepa Kasinathan ${ }^{5}$, Jan Rusz ${ }^{6}$, Peter Schattschneider ${ }^{3,7}$, Ludwig Schultz ${ }^{1,2}$ and Bernd Rellinghaus ${ }^{1}$

1. IFW Dresden, Institute for Metallic Materials, Dresden, Germany

2. TU Dresden, Institut für Festkörperphysik, Dresden, Germany

3. Vienna University of Technology, USTEM, Vienna, Austria

4. McMaster University, Department of Materials Science and Engineering, Hamilton, Canada

5. MPI CPfS, Department Physics of Correlated Matter, Dresden, Germany

6. Uppsala University, Department of Physics and Astronomy, Uppsala, Sweden

7. Vienna University of Technology, Institute of Solid State Physics, Vienna, Austria

Electron energy-loss magnetic chiral dichroism (EMCD), which is the electron wave analogue of X-ray magnetic circular dichroism (XMCD), offers the possibility to study magnetic properties at the nanoscale in a transmission electron microscope (TEM). The relatively young method of EMCD [1] was already refined to such an extent that it is possible to probe magnetic moments of thin films of a variety of ferromagnets $[2,3,4]$. Meanwhile, these measurements surpass the resolution of XMCD experiments. However, quantitative EMCD measurements are so far only reported on thin films rather than on nanoparticles, which are expected to reveal distinct magnetic properties due to their reduced dimensions and enhanced surface to volume ratio.

We report on EMCD measurements on a single FePt nanoparticle (cf. Figure 1) and compare our experimental findings with simulations. $\mathrm{L} 1_{0}$ ordered $\mathrm{FePt}$ is a particularly interesting material since it offers the highest magneto-crystalline anisotropy among the oxidation-resistant hard magnets [5]. It is therefore a promising materials candidate for future high density magnetic data storage media. $\mathrm{L} 1_{0}$ ordered FePt nanoparticles on a STO substrate are prepared by sputtering. Prior to the spectroscopic measurements, samples in plan view geometry are subjected to mechanical thinning and grazing incidence $\mathrm{Ar}^{+}$ion milling in order to obtain substrate-free nanoparticles.

The dichroic signals at the $\mathrm{Fe} \mathrm{L}_{3}$ and $\mathrm{L}_{2}$ edges are expected to amount to no more than $10 \%$ of the absorption edge intensity. Due to these small intensity differences in the electron energy loss (EEL) spectra, a proper microscope alignment, a good reproducibility, a sufficiently stable sample, and a decent signal-to-noise $(\mathrm{S} / \mathrm{N})$ ratio pose extreme experimental demands. The experiments were performed on a FEI Titan ${ }^{3}$ 80-300 microscope equipped with an image $\mathrm{C}_{\mathrm{S}}$ corrector. The sample was oriented under three beam conditions with the [001] easy axis of L10 FePt oriented (close to) parallel to the electron beam. Binned-gain acquisition of the EEL spectra was used to optimize the S/N ratio [6]. Particular attention was paid to the analysis of the EEL spectra. A measurement route is presented that allows for the extraction of a dichroic signal from spectra that still suffer from non-optimal $\mathrm{S} / \mathrm{N}$ ratio. Our experiments are supported by simulations of EEL spectra utilizing the WIEN2k program package [7] in combination with Bloch-wave (BW) methods. These simulations are used to (pre-)determine optimal experimental parameters, that provide for the highest EMCD signals [8, 9]. The experiments reveal a small but reproducible dichroic signal (cf. Figure 2) that agrees well with the results of the theoretical calculations. From these experimental spectra, a ratio of angular to spin moment $\mathrm{m}_{\mathrm{l}} / \mathrm{m}_{\mathrm{s}}=$ $0.084 \pm 0.076$ is for the first time quantitatively derived for individual FePt nanoparticles, which agrees well with the XMCD result $\mathrm{m}_{\mathrm{l}} / \mathrm{m}_{\mathrm{s}}=0.092$ for a large ensemble of $\mathrm{L} 1_{0}$ ordered FePt nanoparticles [10] $[11,12]$. 


\section{References:}

[1] P. Schattschneider et al, Nature 441 (2006), p. 486.

[2] T. Thersleff et al, Scientific Reports 5 (2015), 13012.

[3] X. Fu et al, Appl. Phys. Lett. 107 (2015), 062402.

[4] D. Song et al, Appl. Phys. Lett. 107 (2015), 042401.

[5] J. Lyubina et al, Handbook of Magnetic Materials 19 (2011), p. 291.

[6] M. Bosman and V. J. Keast, Ultramicroscopy 108 (2008), p. 837.

[7] K. Schwarz and P. Blaha, Computational Materials Science 28 (2003), p. 259.

[8] S. Löffler and P. Schattschneider, Ultramicroscopy 110 (2010), p. 831.

[9] J. Rusz, S. Muto and K. Tatsumi, Ultramicroscopy 125 (2013), p. 81.

[10] C. Antoniak et al, Phys. Rev. Lett. 97 (2006), 117201.

[11] P. Schattschneider acknowledges financial support by the Austrian Science Fund (FWF) under grant nr. I543-N20.

[12] S. Löffler acknowledges financial support by the Austrian Science Fund (FWF) under grant nr. J3732N27.

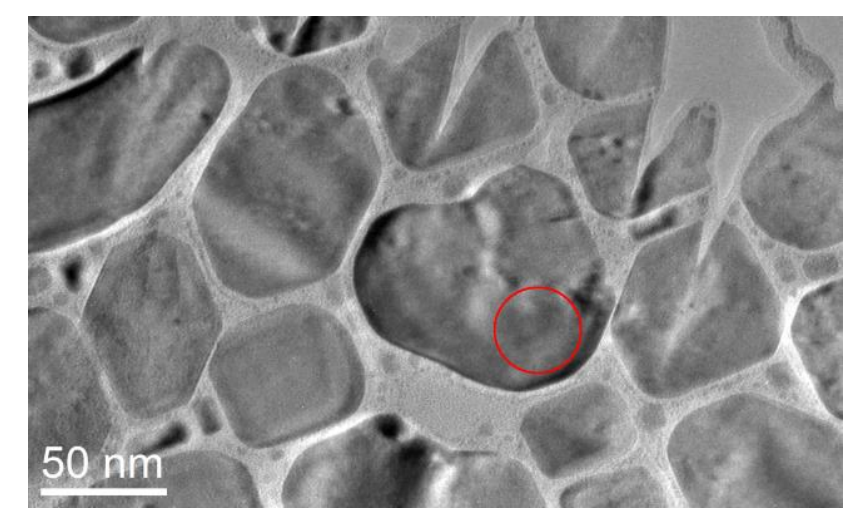

Figure 1. FePt nanoparticles. The area from which the EEL spectra are collected is marked with a red circle.

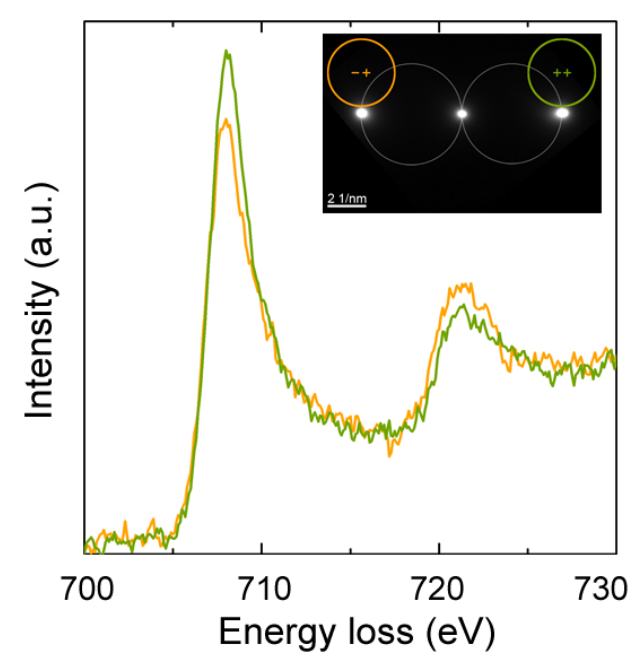

Figure 2. EEL spectra showing a dichroic signal at the $\mathrm{Fe} \mathrm{L}_{3} / \mathrm{L}_{2}$ absorption edges. The inset the diffraction pattern under three beam conditions. The areas, from which the EEL spectra are collected, are marked with circles. 